\title{
More medications, more problems: results from the Sedation Level after Emergent Exlap with Packing for TRAUMA (SLEEP-TRAUMA) study
}

\author{
Tracey Kim ${ }^{1}$. Christopher Celis ${ }^{1}$. Andrew Pop ${ }^{1} \cdot$ Kaitlin McArthur $^{1} \cdot$ Thomas Robert Bushell $^{1} \cdot$ Xian Luo-Owen $^{1}$. \\ Lourdes Swentek $^{1} \cdot$ Sigrid Burruss ${ }^{1} \cdot$ Steven Brooks ${ }^{2} \cdot$ David Turay $^{1} \cdot$ Kaushik Mukherjee $^{1}$
}

Received: 21 May 2020 / Accepted: 6 October 2020 / Published online: 19 October 2020

(c) Springer-Verlag GmbH Germany, part of Springer Nature 2020

\begin{abstract}
Purpose Sedation management of trauma patients after damage control laparotomy (DCL) has not been optimized. We evaluated if shorter sedation exposure was associated with increased proportion of delirium-free/coma-free (DF/CF-ICU) days and change in time to definitive fascial closure (DFC).

Methods We reviewed trauma DCL patients at an ACS-verified level I center over 5 years as shorter (SE) or longer than median (LE) sedation exposure. We compared demographics, injury patterns, hemodynamic parameters, and injury severity between groups. We calculated the propensity for each patient to achieve DFC using age, gender, ISS, red blood cell transfusion, bowel discontinuity, abdominal vascular injury, and time to first takeback; we then determined the effect of sedation exposure on rate of DFC by multivariate Cox regression, adjusted for propensity to achieve DFC. We used linear regression adjusted for age, ISS, head-AIS, bowel discontinuity, and vascular injury to determine the effect of sedation exposure on the proportion of DF/CF-ICU days.

Results 65 patients (33.8\% penetrating) had mean age 41.8 \pm 16.0 , ISS $27.1 \pm 14.2$, Head-AIS $1.2 \pm 1.6$ and median sedation exposure of 2.2 [IQR $0.78,7.3$ ] days (35 SE and $30 \mathrm{LE}$ ). Pattern and severity of solid organ injuries and proportion of small and large bowel and vascular injuries were similar between groups. LE had more abdominal sepsis (23.3\% vs $0 \%$, $p=0.003)$ and enterocutaneous fistula $(16.7 \%$ vs $0 \%, p=0.016)$, and more ventilator $(17.3 \pm 12.7$ vs $6.1 \pm 6.8, p<0.001)$, ICU $(20.8 \pm 14.2$ vs $7.2 \pm 7.6, p<0.001)$, and hospital days (29.6 \pm 19.6 vs $13.9 \pm 9.0, p<0.001)$. DFC was achieved more rapidly in the $\mathrm{SE}$ group $(2.0 \pm 1.5$ days vs $3.9 \pm 3.7$ days [unadjusted], $p=0.023)$ and $\mathrm{SE}$ had a higher proportion of unadjusted DF/CF-ICU days $(33.0 \pm 32.0 \%$ vs $18.1 \pm 16.4 \%, p=0.020)$.

SE was associated with an increased proportion of adjusted DF/CF-ICU days by multivariate linear regression (13.1\% [95\% CI 1.4-24.8\%], $p=0.029$ ) and with faster adjusted rate of DFC by multivariate Cox regression (RR 2.28 [95\% CI 1.25-4.15, $p=0.007])$.

Conclusions Shorter sedation exposure is associated with increased proportion of DF/CF-ICU days and more rapid DFC after DCL for trauma.
\end{abstract}

Keywords Trauma $\cdot$ Damage control laparotomy $\cdot$ Sedation $\cdot$ Delirium $\cdot$ Definitive fascial closure

List of meetings presented: SCCM Feb 2019, San Diego, CA; Southern CA ACS Jan 2019, Santa Barbara, CA.

Electronic supplementary material The online version of this article (https://doi.org/10.1007/s00068-020-01524-9) contains supplementary material, which is available to authorized users.

Kaushik Mukherjee

kmukherjee@1lu.edu

Extended author information available on the last page of the article

\section{Background}

Damage control laparotomy (DCL) is frequently used for trauma and general surgical emergencies. This technique allows time for correction of coagulopathy, hypothermia, and acidosis to improve survival [1-3]. Definitive fascial closure (DFC) is ideal after resuscitation and completion of surgery. Failure to achieve DFC expediently is associated with increased incidence of wound infections, enterocutaneous fistulae, intra-abdominal abscess, and ventral hernia [4-6]. These morbidities dramatically increase the longer 
the abdomen is open, particularly after 8 days [4]. American Association for the Surgery of Trauma (AAST) multicenter studies indicate that decreasing time to "second look laparotomy" and subsequent laparotomies enabled more rapid definitive fascial closure $[7,8]$.

Interventions such as diuretic administration, deep sedation, and chemical paralysis have also been previously endorsed in older literature to help achieve DFC more rapidly [9-12]. However, recent literature has failed to support that these interventions promote more rapid DFC. The group from Vanderbilt found that furosemide infusion was not effective in improving time to DFC [13]. They also published data indicating that chemical paralysis did not affect time to DFC after trauma DCL, with no significant difference in mortality [14]. Additionally, their study showed that patients with neuromuscular blockade had higher sedation requirements [15].

Deep sedation and chemical paralysis have been associated with a high incidence of delirium in critically ill patients, increased mortality, prolonged cognitive dysfunction, and psychiatric conditions [16-24]. There is a paucity of data exploring the specific impacts of sedation and delirium after trauma DCL. Currently, no practice consensus exists regarding sedation strategies in this patient population, and in some centers patients may even be extubated before DFC [25].

Survey data from the Sedation Level after Emergency Exlap with Packing (SLEEP) survey conducted of the Eastern Association for the Surgery of Trauma (EAST) membership indicated that use of sedation and paralytics correlated with the amount of time attending surgeons had been out of residency or fellowship. More experienced surgeons were more likely to sedate deeply, use paralytics, and wait longer for the first and subsequent takeback laparotomies. These findings demonstrate that the current practice pattern after trauma DCL may be experience based rather than evidence based [26].

The authors reviewed the experience in the post-trauma DCL population at a single ACS-verified adult level I trauma center over a 5-year period, focusing on the endpoints of proportion of delirium-free coma-free (DF/CF-ICU) days and the rate of achieving DFC.

\section{Methods}

\section{Inclusion and exclusion criteria}

We reviewed the trauma registry at a single ACS-verified level I adult trauma center from 2014 to 2018. This study was exempt from IRB review. We included adults undergoing trauma DCL. Patients younger than 18 years, pregnant women, prisoners and patients who died before the first takeback operation were excluded.

\section{Surgical and anesthetic management}

Indications for DCL included bleeding injuries requiring packing, extreme visceral or retroperitoneal swelling, vascular or gastrointestinal injuries that required a second look, major abdominal wall tissue loss, or poor condition of fascia [27]. Furthermore, physiological criteria included acidosis $(\mathrm{pH}<7.2$ ), hypothermia less than 34 degrees Centigrade, massive transfusion defined as $>10$ units PRBC, persistent shock, or coagulopathy [12]. Management was left at the discretion of the attending surgeon and anesthesiologist. Our first-line management for patients with DCL includes negative pressure wound therapy with a commercially available device (AbThera, 3M-KCI Inc., St. Paul $\mathrm{MN}$ ). One of our attending surgeons typically constructs an individualized negative pressure wound therapy device that utilizes a thoracostomy tube to deliver suction and an iodine impregnated adherent dressing for achieving a seal (Ioban, 3M Inc., St. Paul MN) as an alternative to the commercially available device. Patients who are felt at the discretion of the attending surgeon to likely require multiple operative interventions to achieve closure undergo placement of a hook and loop-based fabric sheet device that is sutured to the fascia with running polydiaxanone suture (Wittmann Patch, Starsurgical Inc., Burlington WI). This device is then sequentially tightened, either at the bedside in the intensive care unit or in the operating room. If the entire fascia cannot be closed at one time, the one of the above techniques is used to reestablish negative pressure wound therapy until the patient can be returned to the operating room. At our institution, critically injured patients with an open abdomen are typically left intubated and transported to the surgical intensive care unit, where they are reversed upon arrival and spontaneous awakening and breathing trials are performed daily $[16,18]$.

\section{Critical care management}

Our surgical ICU is staffed by anesthesia and surgical intensivists. Propofol and dexmedetomidine are standard first-line sedation agents, and choice of agent was per the discretion of the intensivist. Daily spontaneous awakening and breathing trials were performed daily for patients on continuous intravenous sedation $[16,18,28]$. At our institution, an open abdomen is not considered a contraindication to extubation $[25,29]$. During the time period covered by this study, we did not have an established early mobilization pathway for patients with damage control laparotomy. 


\section{Data collection}

All patients had clinical and demographic data collected including age, gender, mechanism, injury severity score (ISS), head anatomic injury score (Head-AIS), presence of small bowel, colon, and abdominal vascular injuries, type of temporary abdominal closure, time to DFC, and duration of sedative infusion exposure (propofol, dexmedetomidine, and benzodiazepines). Patients were classified based on shorter than median (SE) or longer than median (LE) sedation exposure. The entire exposure to sedation while in the ICU was recorded as patients were still at risk for ICU delirium even after DFC.

Primary endpoints were the rate of achieving DFC and the proportion of DF/CF-ICU days, defined as the number of ICU days during which the Richmond Agitation Sedation Score (RASS) was at least -3 and the Confusion Assessment Method for the ICU (CAM-ICU) score was negative divided by the total number of ICU days for each patient and converted to a percentage. Dehiscence was defined as a separation of the abdominal wall fascia noted on exam and occurring acutely during the index hospitalization. Evisceration was defined as extruded abdominal contents visible on exam. Abdominal sepsis was defined as the presence of tachycardia, hypotension, or altered mental status due to infection with a clinically attributed abdominal source.

\section{Statistical analysis}

Standard parametric methods were used. The median length of sedation exposure was determined a priori to be the splitting point between the SE and LE groups. Linear regression was utilized to determine the effect of age, ISS, Head-AIS, bowel discontinuity, abdominal vascular injury, and short or long sedation exposure on the proportion of DF/CF-ICU days.

To determine the adjusted effect of SE vs LE on the rate of achieving definitive fascial closure, we first calculated a propensity score for each patient in the study to achieve DFC based on age, gender, ISS, packed red blood cells transfused, bowel discontinuity, abdominal vascular injury, and time to first takeback laparotomy. The propensity score was then used as a covariate in a Cox regression used to determine the adjusted effect of SE vs LE on the rate of achieving DFC [30].

\section{Results}

\section{Demographics}

Demographic data for our 65-patient cohort are shown in Table 1. Median length of sedation was 2.2 [IQR 0.78, 7.3] days and the mean length of sedation in each group was $1.0 \pm 1.0$ days in the SE group versus $12.6 \pm 10.6$ days in the LE group $(p<0.001) .35$ patients were in the SE group and 30 patients were in the LE group. Mean age, gender distribution, ISS, Head-AIS, and BMI were not different between the two groups. The rate of penetrating injury was similar between groups (42.9\% in the SE group and $33.3 \%$ in the LE group, $p=0.619)$. Initial temperature, respiratory rate, and GCS were not different between groups. Initial heart rate was higher in the LE group $(118.4 \pm 22.6$ versus $97.5 \pm 30.1 \mathrm{bpm}$, $p=0.005$ ), but systolic blood pressure was not significantly different. The type and pattern of solid organ injuries was not different between groups.

The injury burden of both groups was similar, with mean ISS $25.2 \pm 14.9$ (SE) and 29.3 $\pm 13.2(\mathrm{LE}, p=0.241$ ). Head-AIS was similar between the SE $(1.1 \pm 1.6)$ and LE $(1.4 \pm 1.6)$ groups $(p=0.574)$. The proportion of patients with abdominal vascular injury, small bowel injury, and colon injury was not different between groups. The proportion of patients with small bowel resection, colon resection, intestinal discontinuity, or intentionally retained surgical packing was not different between groups. A commercially available negative pressure wound therapy device was used in 59/65 patients (90.7\%), an improvised negative pressure wound therapy device was used in $6 / 65(9.2 \%)$ patients, and a Velcro-based sequential tightening device was used in $7 / 66$ (10.8\%) patients, with no differences between groups.

The LE group had a higher transfusion requirement with respect to PRBC but not FFP $(23.1 \pm 24.9$ vs. $9.6 \pm 9.3$ units PRBC, $p=0.008$ and $9.8 \pm 14.7$ vs $4.4 \pm 5.7$ units FFP, $p=0.064)$. Fluid balance from admission to DFC was more positive in the LE group, although not significantly $(8.3 \pm 8.4$ vs $5.4 \pm 5.4 \mathrm{~L}, p=0.153)$.

\section{Outcomes and complications}

Outcomes and complications are demonstrated in Table 2. The rate of achieving DFC was similar between groups with $94.4 \%(34 / 36)$ in the SE group and $83.3 \%(25 / 30)$ in the LE group $(p=0.087)$. By the time two takebacks had occurred, the SE group had a significantly higher proportion of patients having achieved DFC. This was maintained after three and after four or more takebacks. All surviving patients in the SE group were closed by the fourth takeback. The patient in the SE group who did not achieve DFC died due to recalcitrant hemorrhage and coagulopathy with an open abdomen. In the LE group, two patients were discharged to hospice with a chronically open abdomen in the setting of multiorgan failure and recurrent sepsis. Three additional patients were closed with polyglycolic acid/trimethylene carbonate hybrid mesh (Bio-A ${ }^{\circledR}$, WL Gore \& Associates, Inc., Flagstaff, AZ). Time to DFC was longer in the LE group (3.9 \pm 3.7 days 
Table 1 Patient characteristics

\begin{tabular}{|c|c|c|c|}
\hline & $\begin{array}{l}\text { Short exposure }(N=35) \\
\text { Mean } \pm \text { Std Dev or } N(\%)\end{array}$ & $\begin{array}{l}\text { Long exposure }(N=30) \\
\text { Mean } \pm \text { Std Dev or } N(\%)\end{array}$ & $P$ value \\
\hline$\%$ Male & $29 / 35(82.9 \%)$ & $19 / 30(63.3 \%)$ & 0.094 \\
\hline$\%$ Penetrating Trauma & $15 / 35(42.9 \%)$ & $10 / 30(33.3 \%)$ & 0.619 \\
\hline Age (years) & $39.4 \pm 14.8$ & $44.6 \pm 17.0$ & 0.196 \\
\hline 6 & $36.1 \pm 0.9$ & $36.3 \pm 0.5$ & 0.552 \\
\hline Initial Respiratory Rate (breaths/min) & $21.9 \pm 5.7$ & $25.5 \pm 11.2$ & 0.168 \\
\hline Initial Heart Rate (beats/min) & $97.5 \pm 30.1$ & $118.4 \pm 22.6$ & 0.005 \\
\hline Initial Systolic Blood Pressure (mm Hg) & $104.9 \pm 30.9$ & $116.7 \pm 30.2$ & 0.166 \\
\hline Initial Glasgow Coma Scale (GCS) & $10.2 \pm 5.1$ & $10.8 \pm 4.8$ & 0.652 \\
\hline Injury Severity Score (ISS) & $25.2 \pm 14.9$ & $29.3 \pm 13.2$ & 0.241 \\
\hline Head Anatomic Injury Score (AIS) & $1.1 \pm 1.6$ & $1.4 \pm 1.6$ & 0.574 \\
\hline Body mass index $\left(\mathrm{kg} / \mathrm{m}^{2}\right)$ & $28.6 \pm 5.5$ & $29.4 \pm 7.8$ & 0.656 \\
\hline Abdominal Vascular Injury & $10 / 35(28.6 \%)$ & $8 / 30(26.7 \%)$ & 1.000 \\
\hline Small Bowel Injury & $15 / 35(42.9 \%)$ & $9 / 30(30.0 \%)$ & 0.314 \\
\hline Colon Injury & $8 / 35(22.9 \%)$ & $10 / 30(33.3 \%)$ & 0.253 \\
\hline Liver Laceration (AAST Grading) & & & 0.086 \\
\hline None & $16 / 35(45.7 \%)$ & $18 / 30(60.0 \%)$ & \\
\hline I & $1 / 35(2.9 \%)$ & $4 / 30(13.3 \%)$ & \\
\hline II & $5 / 35(14.3 \%)$ & $0 / 30(0.0 \%)$ & \\
\hline III & $9 / 35(25.7 \%)$ & $4 / 30(13.3 \%)$ & \\
\hline IV & $4 / 35(11.4 \%)$ & $3 / 30(10.0 \%)$ & \\
\hline $\mathrm{V}$ & $0 / 35(0.0 \%)$ & $1 / 30(3.3 \%)$ & \\
\hline Spleen Laceration (AAST Grading) & & & 0.645 \\
\hline None & $28 / 35(80.0 \%)$ & $21 / 30(70.0 \%)$ & \\
\hline I & $2 / 35(5.7 \%)$ & $4 / 30(13.3 \%)$ & \\
\hline II & $3 / 35(8.6 \%)$ & $2 / 30(6.7 \%)$ & \\
\hline III & $0 / 35(0.0 \%)$ & $0 / 30(0.0 \%)$ & \\
\hline IV & $2 / 35(5.7 \%)$ & $2 / 30(6.7 \%)$ & \\
\hline $\mathrm{V}$ & $0 / 35(0.0 \%)$ & $1 / 30(3.3 \%)$ & \\
\hline Renal Laceration (AAST Grading) & & & 0.973 \\
\hline None & $31 / 35(88.6 \%)$ & $27 / 30(90.0 \%)$ & \\
\hline I & $0 / 35(0.0 \%)$ & $0 / 30(0.0 \%)$ & \\
\hline II & $1 / 35(2.9 \%)$ & $1 / 30(3.3 \%)$ & \\
\hline III & $0 / 35(0.0 \%)$ & $0 / 30(0.0 \%)$ & \\
\hline IV & $2 / 35(5.7 \%)$ & $1 / 30(3.3 \%)$ & \\
\hline $\mathrm{V}$ & $1 / 35(2.9 \%)$ & $1 / 30(3.3 \%)$ & \\
\hline Pancreatic Laceration (AAST Grading) & & & 0.548 \\
\hline None & $34 / 35(97.1 \%)$ & $28 / 30(93.3 \%)$ & \\
\hline I & $0 / 35(0.0 \%)$ & $0 / 30(0.0 \%)$ & \\
\hline II & $1 / 35(2.9 \%)$ & $1 / 30(3.3 \%)$ & \\
\hline III & $0 / 35(0.0 \%)$ & $1 / 30(3.3 \%)$ & \\
\hline IV & $0 / 35(0.0 \%)$ & $0 / 30(0.0 \%)$ & \\
\hline $\mathrm{V}$ & $0 / 35(0.0 \%)$ & $0 / 30(0.0 \%)$ & \\
\hline Small Bowel Resection at First OR & $12 / 35(34.3 \%)$ & $6 / 30(20.0 \%)$ & 0.269 \\
\hline Colon Resection at First OR & $6 / 35(17.1 \%)$ & $8 / 30(26.7 \%)$ & 0.264 \\
\hline Packs Left in Abdomen at First OR & $26 / 35(74.3 \%)$ & $23 / 30(76.7 \%)$ & 1.000 \\
\hline Bowel Discontinuity at First OR & $7 / 35(20.0 \%)$ & $9 / 30(30.0 \%)$ & 0.259 \\
\hline Total PRBC (units) & $9.6 \pm 9.3$ & $23.1 \pm 24.9$ & 0.008 \\
\hline Total FFP (units) & $4.4 \pm 5.7$ & $9.8 \pm 14.7$ & 0.064 \\
\hline Fluid balance from admission until DFC (L) & $5.4 \pm 5.4$ & $8.3 \pm 8.4$ & 0.153 \\
\hline
\end{tabular}

Bolded numbers indicate $p$ values with statistical significance 
Table 2 Outcomes

\begin{tabular}{lllr}
\hline & $\begin{array}{l}\text { Short exposure }(N=35) \\
\text { Mean } \pm \text { Std Dev or } N(\%)\end{array}$ & $\begin{array}{l}\text { Long exposure }(N=30) \\
\text { Mean } \pm \text { Std Dev or } N(\%)\end{array}$ & $P$ value \\
\hline DFC Achieved & $34 / 35(97.1 \%)$ & $25 / 30(83.3 \%)$ & 0.087 \\
After 1 takeback & $24 / 35(68.5 \%)$ & $14 / 30(46.7 \%)$ & 0.084 \\
After 2 takebacks & $31 / 34(91.2 \%)$ & $17 / 30(56.7 \%)$ & $\mathbf{0 . 0 0 3}$ \\
After 3 takebacks & $33 / 34(97.1 \%)$ & $21 / 30(70.0 \%)$ & $\mathbf{0 . 0 0 4}$ \\
After 4 or more takebacks & $34 / 34(100.0 \%)$ & $25 / 30(83.3 \%)$ & $\mathbf{0 . 0 1 9}$ \\
Time to DFC (days) & $2.0 \pm 1.5$ & $3.9 \pm 3.7$ & $\mathbf{0 . 0 2 3}$ \\
Proportion of Delirium-free/coma- & $33.0 \pm 32.0$ & $18.1 \pm 16.4$ & $\mathbf{0 . 0 2 0}$ \\
free (DF/CF-ICU) days $(\%)$ & & & $<\mathbf{0 . 0 0 1}$ \\
Total ventilator days & $6.1 \pm 6.8$ & $17.3 \pm 12.7$ & $<\mathbf{0 . 0 0 1}$ \\
Total ICU days & $7.2 \pm 7.6$ & $20.8 \pm 14.2$ & $<\mathbf{0 . 0 0 1}$ \\
Hospital LOS (days) & $13.9 \pm 9.0$ & $29.6 \pm 19.6$ & 0.230 \\
Wittmann patch used & $2 / 35(5.7 \%)$ & $5 / 29(17.2 \%)$ & 0.199 \\
Unplanned return to OR & $4 / 35(11.4 \%)$ & $8 / 30(26.7 \%)$ & 0.209 \\
Dehiscence/evisceration & $0 / 35(0 \%)$ & $2 / 30(6.7 \%)$ & $\mathbf{0 . 0 0 3}$ \\
Abdominal sepsis & $0 / 35(0 \%)$ & $7 / 30(23.3 \%)$ & $\mathbf{0 . 0 1 7}$ \\
Enterocutaneous fistula & $0 / 35(0 \%)$ & $5 / 30(16.7 \%)$ & 0.239 \\
Pneumonia & $10 / 35(28.6 \%)$ & $12 / 30(40.0 \%)$ & 0.400 \\
Survival to discharge & $31 / 35(88.6 \%)$ & $25 / 30(83.3 \%)$ & $\mathbf{0 . 0 2 3}$ \\
Discharge to home & $17 / 35(48.6 \%)$ & $5 / 30(16.7 \%)$ & \\
\hline & & & \\
\hline
\end{tabular}

Bolded numbers indicate $p$ values with statistical significance

vs $2.0 \pm 1.5$ days, $p=0.023)$. The proportion of $\mathrm{DF} / \mathrm{CF}-$ ICU days was higher in the SE group $(33.0 \% \pm 32.0 \%$ vs $18.1 \% \pm 16.4 \%, p=0.020)$. Ventilator days $(17.3 \pm 12.7$ vs $6.1 \pm 6.8, p<0.001)$, ICU days $(20.8 \pm 14.2$ vs $7.2 \pm 7.6$, $p<0.001)$, and hospital days $(29.6 \pm 19.6$ vs $13.9 \pm 9.0$, $p<0.001)$ were all longer in the LE group.

$26.7 \%$ (8/30) LE patients vs $13.9 \%$ (5/36) SE patients had unplanned return to OR, although this was not statistically significant. Seven patients (four in the SE group and three in the LE group) were re-explored for bleeding. One SE patient was re-explored due to concern for dehiscence but this was not seen at laparotomy. Two patients in the LE group were re-explored twice, once each for bleeding and abdominal sepsis due to anastomotic leak. One LE patient was explored for evisceration, one for abdominal compartment syndrome caused by necrotic colon and terminal ileum, and one for anastomotic breakdown.

Rates of abdominal sepsis (23.3\% vs $0 \%, p=0.003$ ) and enterocutaneous fistula ( $16.7 \%$ vs $0 \%, p=0.017)$ were higher in the LE group, although dehiscence and evisceration were similar $(6.7 \%$ vs $0 \%, p=0.209)$ between groups. Incidence of pneumonia was comparable between groups. Of the two patients with dehiscence and/or evisceration, one died without achieving secondary fascial closure and one was closed with retention sutures. Although a similar number of patients in both groups survived to discharge, only $16.7 \%$ (5/30) of the LE patients were discharged to home as compared to $48.6 \%(17 / 35)$ of the SE patients $(p=0.018)$.

\section{Critical care management}

Similar proportions of patients were treated with fentanyl infusions in the LE and SE groups (96.7\% [29/30] vs 86.1\% [31/36], $p=0.209$ ) but the LE patients had more infusion days $(9.3 \pm 8.9$ vs $2.0 \pm 2.2, p<0.001)$ (Table 3$)$. More patients in the LE group used propofol infusions $(96.7 \%$ [29/30] vs $75.0 \%$ [27/36], $p=0.017)$ and were treated for more days $(6.0 \pm 5.0$ vs $0.85 \pm 0.92, p<0.001)$. More patients in the LE group also used dexmedetomidine infusions $(90.0 \%$ [27/30] vs $22.2 \%$ [8/36], $p<0.001)$ and had more infusion days $(6.3 \pm 8.6$ vs $0.17 \pm 0.42, p=0.001)$. Use of benzodiazepine infusions was uncommon in both the LE and SE groups $(16.7 \%$ [5/30] vs $2.8 \%[1 / 36], p=0.084)$ and for a short period in both groups (SE: $0.01 \pm 0.04 \mathrm{~d}$, LE: $0.25 \pm 0.69 \mathrm{~d}, p=0.056)$. Only three patients had chemical paralysis used, all in the LE group, for a mean exposure of $1.6 \pm 0.44$ days $(p<0.001)$. Two patients in the SE group and four patients in the LE group were extubated prior to DFC.

\section{Linear regression analysis}

Proportion of DF/CF-ICU days, adjusted for age, ISS, HeadAIS, bowel discontinuity, and abdominal vascular injury, was increased in the SE group by $13.1 \%$ [95\% CI $1.4 \%$, 
Table 3 Critical care management

\begin{tabular}{lllc}
\hline & $\begin{array}{l}\text { Short exposure }(N=35) \\
\text { Mean } \pm \text { Std Dev or } N(\%)\end{array}$ & $\begin{array}{l}\text { Long exposure }(N=30) \\
\text { Mean } \pm \text { Std Dev or } N(\%)\end{array}$ & $P$ value \\
\hline Fentanyl infusion (\%) & $31 / 35(88.6 \%)$ & $29 / 30(96.7 \%)$ & 0.229 \\
Mean exposure (d) & $2.0 \pm 2.2$ & $9.3 \pm 8.9$ & $<\mathbf{0 . 0 0 1}$ \\
Propofol infusion (\%) & $27 / 35(77.1 \%)$ & $29 / 30(96.7 \%)$ & $\mathbf{0 . 0 2 4}$ \\
Mean exposure (d) & $0.85 \pm 0.92$ & $6.0 \pm 5.0$ & $<\mathbf{0 . 0 0 1}$ \\
Dexmedetomidine infusion (\%) & $8 / 35(22.9 \%)$ & $27 / 30(90.0 \%)$ & $<\mathbf{0 . 0 0 1}$ \\
Mean exposure (d) & $0.17 \pm 0.42$ & $6.3 \pm 8.6$ & $\mathbf{0 . 0 0 1}$ \\
Benzodiazepine infusion (\%) & $1 / 35(2.9 \%)$ & $5 / 30(16.7 \%)$ & 0.068 \\
Mean exposure (d) & $0.01 \pm 0.04$ & $0.26 \pm 0.69$ & 0.056 \\
Any sedation infusion & $30 / 35(85.7 \%)$ & $30 / 30(100.0 \%)$ & 0.057 \\
Mean exposure (d) & $1.0 \pm 1.0$ & $12.6 \pm 10.6$ & $<\mathbf{0 . 0 0 1}$ \\
Paralytic infusion (\%) & $0 / 35(0 \%)$ & $3 / 30(10 \%)$ & 0.0923 \\
Mean exposure (d) & $0.0 \pm 0.0$ & $1.6 \pm 0.44$ & $<\mathbf{0 . 0 0 1}$ \\
Extubated prior to DFC (\%) & $2 / 35(5.7 \%)$ & $4 / 30(13.3 \%)$ & 0.413 \\
\hline
\end{tabular}

Bolded numbers indicate $p$ values with statistical significance

\begin{tabular}{llc}
\hline Covariate & $\begin{array}{l}\text { Effect on DF/CF-ICU days } \\
\% \text { change }[95 \% \mathrm{CI}]\end{array}$ & $P$ value \\
\hline Age (per decade) & $-3 \%[-7 \%,+1 \%]$ & 0.207 \\
Injury Severity Score (ISS) & $-0.2 \%[-0.7 \%,+0.2 \%]$ & 0.289 \\
Head Anatomic Injury Score (AIS) & $\mathbf{- 4 . 6 \%}[-\mathbf{0 . 3} \%, \mathbf{- 8 . 9 \%}]$ & $\mathbf{0 . 0 3 8}$ \\
Shorter vs. Longer sedation exposure & $\mathbf{1 3 . 1 \%}[\mathbf{1 . 4 \% , \mathbf { 2 4 . 8 } \%}]$ & $\mathbf{0 . 0 2 9}$ \\
Bowel discontinuity & $\mathbf{1 3 . 8 \%}[\mathbf{0 . 5} \%, \mathbf{2 7 . 1} \%]$ & $\mathbf{0 . 0 4 3}$ \\
Abdominal vascular injury & $-9.9 \%[-22.9 \%, 3.1 \%]$ & 0.131 \\
\hline
\end{tabular}

Bolded numbers indicate $p$ values with statistical significance
Table 4 Results of linear regression for proportion of delirium-free/coma-free (DF/ CF-ICU) days
24.8\%, $p=0.029$, Table 4]. Age and overall ISS were not associated with proportion of DF/CF-ICU days but each point increase in Head-AIS decreased the proportion of DF/CF-ICU days by $4.6 \%$ [95\% CI 0.3\%, 8.9\%, $p=0.038$ ]. Bowel discontinuity was associated with an increase in the proportion of DF/CF-ICU days by $13.8 \%$ [95\% CI $0.5 \%$, $27.1 \%, p=0.043]$.

\section{Multivariate Cox regression analysis}

After calculating a propensity score for each patient to achieve DFC incorporating age, gender, injury severity scale (ISS), red blood cell transfusion, bowel discontinuity, abdominal vascular injury, and time to first takeback laparotomy, we generated an adjusted rate ratio for achieving DFC in the SE and LE groups. The propensity score was highly predictive for attaining DFC (RR 18.25 [95\% CI 1.48, 224.7], $p=0.023$ ). Our multivariate Cox regression analysis indicated that SE was associated with faster adjusted rate of achieving DFC (RR 2.28 [95\% CI 1.25, 4.15, $p=0.007$, Fig. 1]).

\section{Discussion}

DCL is a commonly employed surgical technique used in the critically ill following trauma to allow correction of shock, hypothermia and acidosis before definitive repair and primary closure. Completion of DFC is ideal to prevent major complications associated with an open abdomen. In the known English literature, there is no consensus regarding level of sedation and paralysis in the management of open abdomen patients, with some authors recommending deep sedation or even neuromuscular blockade [5, 9-11]. Additionally, actual practice patterns are both disparate and dependent on individual provider experience [26]. Sedation management is key in the open abdomen population, as numerous publications show improved outcomes in critically ill patients with the tenets of the ABCDEF bundle advocated by the Society for Critical Care Medicine (SCCM) [16-18, 31].

Smith et. al. evaluated the use of neuromuscular blocking agents (NBMA) in 222 patients with open abdomens (125 managed with NMBA and 97 managed without). They found no difference in time to closure, hospital length of stay, ICU 


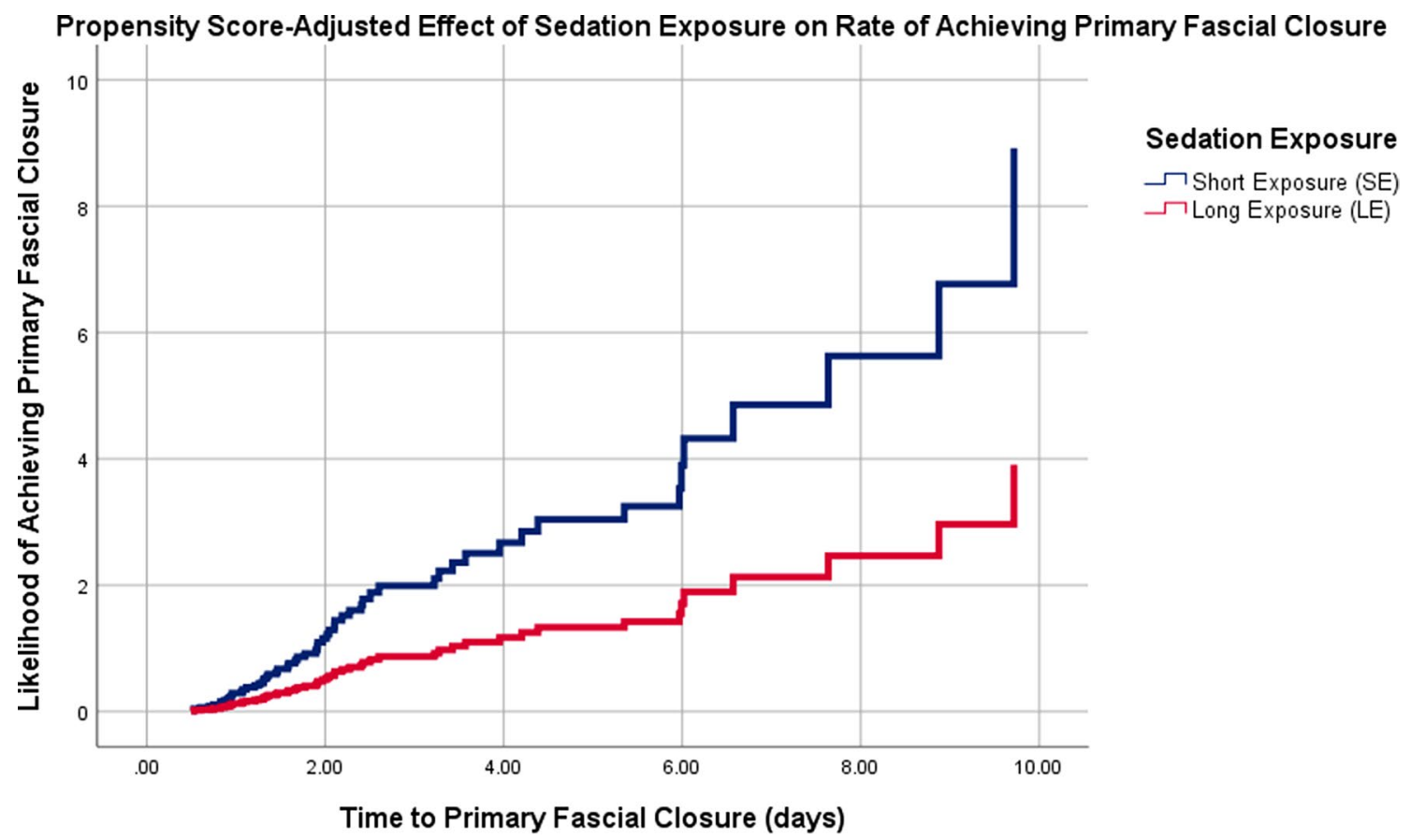

Fig. 1 The propensity score-adjusted rate of achieving definitive fascial closure based on sedation is illustrated above. Propensity for each patient to achieve definitive fascial closure was previously calculated and included age, gender, ISS, RBC transfusion, bowel discontinu-

stay, ventilator-free days and overall mortality [14]. Patients receiving NMBA also required more sedation (typically with propofol) than non-NMBA patients, but not more analgesia (typically with fentanyl). Furthermore, the group noted that increased cumulative propofol dose was associated with a decrease in DF/CF-ICU days, corroborating the information in our study [15]. In our series, only three patients underwent treatment with paralytic infusion, all of whom were in the LE group.

Numerous studies have examined the results of delirium in mechanically ventilated patients. Ely et. al. evaluated a prospective cohort of mechanically ventilated patients, finding that delirium was an independent predictor of higher 6-month mortality and longer hospital stay [17]. In a randomized control trial by Schweickert et. al., sedated and mechanically ventilated adult ICU patients were randomized to early mobilization and daily interruption of sedation versus standard care. Those in the intervention arm had shorter duration of delirium, increased ventilator free days, and improved functional outcome at discharge [32]. Delirium promotes worse long term cognitive outcomes and increased overall mortality [31, 32]. However, this has not been studied in the open-abdomen patient population.

The active and senior EAST membership was surveyed regarding open abdomen management. Surgeon experience (defined as the length of time since the completion ity, abdominal vascular injury, and time to first takeback is illustrated above. This indicates that patients with shorter sedation exposure achieve definitive fascial closure more rapidly than those with longer sedation exposure (RR 2.28 [95\% CI 1.25, 4.15, $p=0.007]$ )

of fellowship or residency) was identified as a key factor in the attitude toward sedation and paralysis. Surgeon experience was more likely associated with increased deep sedation (RASS of -3 to -5 ) and chemical paralysis. Experienced surgeons were less concerned about delirium and more concerned about evisceration as the reason for sedation level choice, and more concerned about evisceration as their indication for paralytics. Using multivariate logistic regression, surgeon experience was associated with increased deep sedation. Trauma center level was also significant, with level III/IV centers being greater than seven times more likely to use deep sedation than level I/II centers $(p=0.008)$. This demonstrates disparate sedation strategies among practicing surgeons, in the face of growing evidence that minimizing sedation can prevent delirium and improve outcomes [26]. Additionally, there is increasing trauma literature supporting extubation prior to DFC, a practice we have adopted in two SE and four LE patients in our series [25].

Our study adds two important contributions to the known literature. First, shorter sedation exposure in critically injured patients after DCL is associated with increased proportion of DF/CF-ICU days. Based on the expected ICU length of stay for this cohort, this corresponds to between 1 and 2 delirium-free coma-free days. Longer duration of delirium has been associated with increased hospital mortality, ICU length of stay, and hospital length of stay in 
postsurgical critically ill patients [33]. In lightly sedated, mechanically ventilated critically ill patients, the additional mortality risk of delirium increased with each additional delirium day, so saving even a single delirium day reduces mortality [34]. This study endorses that one way to accomplish this in trauma patients might be to reduce sedation exposure.

Second, this is the first study in the known English literature that demonstrates shorter sedation exposure is associated with more rapid achievement of definitive fascial closure (RR 2.28 [95\% CI 1.25, 4.15, $p=0.007]$ ), even after adjusting for the propensity to achieve DFC (including age, gender, ISS, RBC transfusion, bowel discontinuity, abdominal vascular injury, and time to first takeback). Shorter sedation exposure patients were also more likely to be discharged home rather than a facility. The study is congruent with other literature advocating for decreased paralysis and lighter sedation.

There are several limitations to this study. This is a retrospective study that does not randomize the length of sedation. The length of sedation was left to the discretion of the provider and may have extended past the time of DFC, particularly if the patient was still mechanically ventilated. Some factors, including severe traumatic brain injury and severe acute hypoxic respiratory failure, may predispose management toward longer sedation exposure. In our study, the mean head AIS score was nearly identical between the two groups, so the effect of severe TBI would be unlikely to introduce bias. The length of mechanical ventilation was longer in the LE group, so this may be related to the length of sedation, although causation is unclear. In general, the vital signs for the two groups were similar at presentation, although there was a higher heart rate in the LE group. There was also a lower initial systolic blood pressure in the LE group, although this was not statistically significant. The injury patterns between the two groups were similar, with no statistically significant differences in the incidence of small bowel injuries, colon injuries, or abdominal vascular injuries. Furthermore, the incidence and AAST grade of solid organ injuries (liver, spleen, kidney, pancreas) was not different between groups. Despite the lack of apparent differences between the SE and LE groups, the fluid balance was more positive in the LE group and the transfusion requirement was higher as well, leading to the inclusion of PRBC transfusion in the Cox regression for time to DFC. It is not immediately clear why the LE group received more resuscitation than the SE group given the similar ISS, headAIS, and injury pattern. Given the nature of the study, it is difficult to differentiate whether patients were given less sedation, because they were closed earlier or if the provider chose to give shorter sedation in those with open abdomens. However, these factors were adjusted for in the course of the regression analysis.
This study demonstrates two novel observations: 1) reduced sedation is associated with a greater delirium-free/ coma-free ICU days in critically injured patients after DCL; 2 ) reduced sedation is associated with more rapid achievement of DFC in this population.

Author's contributions AP: study formulation, data analysis. TB: study formulation, obtaining drug exposure data, critical review of manuscript. KM study formulation, data analysis, statistics, writing/editing manuscript. TK: Writing manuscript. CC, XL, SEB, DT, LS, SB: critical review of manuscript.

Funding None.

Data availability Source data may be obtained by contacting the corresponding author.

\section{Compliance with ethical standards}

Conflict of interest The authors declare that they have no conflict of interest.

Ethics approval Deemed exempt from review by Loma Linda University Medical Center IRB.

Consent to participate Consent requirement waived by Loma Linda University Medical Center IRB.

Consent to publication As the corresponding author, I officially declare that all listed authors have contributed to the manuscript, are aware of its impending submission, and consent to said submission.

\section{References}

1. Rotondo MF, Schwab CW, McGonigal MD, Phillips GR, Fruchterman TM, Kauder DR, et al. "Damage control": an approach for improved survival in exsanguinating penetrating abdominal injury. J Trauma. 1993;35(3):375-82.

2. Stone HH, Strom PR, Mullins RJ. Management of the major coagulopathy with onset during laparotomy. Ann Surg. 1983;197(5):532-5.

3. Burch JM, Ortiz VB, Richardson RJ, Martin RR, Mattox KL, Jordan GL. Abbreviated laparotomy and planned reoperation for critically injured patients. Ann Surg. 1992;215(5):476-83 ((discussion 483-4)).

4. Miller RS, Morris JA Jr, Diaz JJ Jr, Herring MB, May AK. complications after 344 damage-control open celiotomies. J Trauma. 2005;59(6):1365-74.

5. Miller RS, Morris JA. Abdominal compartment syndrome, damage control, and the post-traumatic open abdomen. In: Asensio J, Trunkey D, editors. Current therapy of trauma and surgical critical care. 1st ed. Elsevier Inc; 2008. p 13.

6. Coccolini F, Ceresoli M, Kluger Y, Kirkpatrick A, Montori G, Salvetti F, et al. Open abdomen and entero-atmospheric fistulae: an interim analysis from the International Register of Open Abdomen (IROA). Injury. 2018;2:1-7 ((Elsevier Ltd)).

7. Bradley MJ. Independent predictors of enteric fistula and abdominal sepsis after damage control laparotomy. JAMA Surg. 2013;148(10):947-8 
8. Pommerening MJ, DuBose JJ, Zielinski MD, Phelan HA, Scalea TM, Inaba K, et al. Time to first take-back operation predicts successful primary fascial closure in patients undergoing damage control laparotomy. Surgery. 2014;156(2):431-8.

9. Godat L, Kobayashi L, Costantini T, Coimbra R. Abdominal damage control surgery and reconstruction: world society of emergency surgery position paper. World J Emerg Surg. 2013;8(1):53-7.

10. Kobayashi L, Coimbra R. Planned re-laparotomy and the need for optimization of physiology and immunology. Eur J Trauma Emerg Surg. 2014;40(2):135-42.

11. Dutton WD, Diaz JJ Jr, Miller RS. critical care issues in managing complex open abdominal wound. J Intensive Care Med. 2011;27(3):161-71.

12. Regner JL, Kobayashi L, Coimbra R. Surgical strategies for management of the open abdomen. World J Surg. 2012;36(3):497-510 ((6 ed. Springer-Verlag)).

13. Webb LH, Patel MB, Dortch MJ, Miller RS, Gunter OL, Collier $\mathrm{BR}$. Use of a furosemide drip does not improve earlier primary fascial closure in the open abdomen. J Emerg Trauma Shock. 2012;5(2):126-30.

14. Smith SE, Hamblin SE, Guillamondegui OD, Gunter OL, Dennis BM. Effectiveness and safety of continuous neuromuscular blockade in trauma patients with an open abdomen: a follow-up study. Am J Surg. 2018;216(3):414-9. https://doi.org/10.1016/j. amjsurg.2018.04.003

15. Smith SE, Hamblin SE, Dennis BM. Effect of neuromuscular blocking agents on sedation requirements in trauma patients with an open abdomen. Pharmacotherapy. 2019;39(3):271-9.

16. Kress JP, Pohlman AS, O'Connor MF, Hall JB. Daily interruption of sedative infusions in critically ill patients undergoing mechanical ventilation. New Eng J Med. 2000;342(20):1471-7 ((Massachusetts Medical Society)).

17. Ely EW, Shintani A, Truman B, Speroff T, Gordon SM, Harrell FE, et al. Delirium as a predictor of mortality in mechanically ventilated patients in the intensive care unit. JAMA. 2004;291(14):1753-62 ((American Medical Association)).

18. Girard TD, Kress JP, Fuchs BD, Thomason JWW, Schweickert WD, Pun BT, et al. Efficacy and safety of a paired sedation and ventilator weaning protocol for mechanically ventilated patients in intensive care (Awakening and Breathing Controlled trial): a randomised controlled trial. Lancet. 2008;371(9607):126-34.

19. Rengel KF, Hayhurst CJ, Pandharipande PP, Hughes CG. Longterm cognitive and functional impairments after critical illness. Anesth Analg. 2019;128(4):772-80.

20. Vasilevskis EE, Chandrasekhar R, Holtze CH, Graves J, Speroff $\mathrm{T}$, Girard TD, et al. The cost of ICU delirium and coma in the intensive care unit patient. Med Care. 2018;56(10):890-7.

21. Devlin JW, Skrobik Y, Gélinas C, Needham DM, Slooter AJC, Pandharipande PP, et al. Clinical practice guidelines for the prevention and management of pain, agitation/sedation, delirium, immobility, and sleep disruption in adult patients in the ICU.
Crit Care Med. 2018;46(9):e825-73. https://doi.org/10.1097/ CCM.0000000000003299.

22. Girard TD, Thompson JL, Pandharipande PP, Brummel NE, Jackson JC, Patel MB, et al. Clinical phenotypes of delirium during critical illness and severity of subsequent long-term cognitive impairment: a prospective cohort study. Lancet Respir Med. 2018;6(3):213-22.

23. Marra A, Pandharipande PP, Patel MB. Intensive care unit delirium and intensive care unit-related posttraumatic stress disorder. Surg Clin North Am. 2017;97(6):1215-35.

24. Kenes MT, Stollings JL, Wang L, Girard TD, Ely EW, Pandharipande PP. Persistence of delirium after cessation of sedatives and analgesics and impact on clinical outcomes in critically ill patients. Pharmacotherapy. 2017;37(11):1357-65.

25. Taveras LR, Imran JB, Cunningham HB, Madni TD, Taarea R, Tompeck A, et al. Trauma and emergency general surgery patients should be extubated with an open abdomen. J Trauma Acute Care Surg. 2018;85(6):1043-7.

26. Choi JH, Luo-Owen X, Brooks SE, Turay D, O'Bosky KR, Mukherjee K. Sedation and paralytic use in open abdomen patients-results from the EAST SLEEP Survey. Surgery. 2019;166(6):1111-6.

27. Chiara O, Cimbanassi S, Biffl W, Leppaniemi A, Henry S, Scalea $\mathrm{TM}$ et al. International consensus conference on open abdomen in trauma. J Trauma Acute Care Surg. 2016;80(1):173-83. https ://doi.org/10.1097/TA.0000000000000882.

28. Ely EW, Baker AM, Dunagan DP, Burke HL, Smith AC, Kelly PT, et al. Effect on the duration of mechanical ventilation of identifying patients capable of breathing spontaneously. New Eng J Med. 1996;335(25):1864-9 ((Massachusetts Medical Society)).

29. Sujka JA, Safcsak K, Cheatham ML, Ibrahim JA. Trauma patients with an open abdomen following damage control laparotomy can be extubated prior to abdominal closure. World J Surg. 2018;42(10):3210-4 ((Springer International Publishing)).

30. D'Agostino RB. Propensity score methods for bias reduction in the comparison of a treatment to a non-randomized control group. Statist Med. 1998;17(19):2265-81 ((John Wiley \& Sons, Ltd)).

31. Barnes-Daly MA, Phillips G, Ely EW. Improving hospital survival and reducing brain dysfunction at seven california community hospitals. Crit Care Med. 2017;45(2):171-8.

32. Schweickert WD, Pohlman MC, Pohlman AS, Nigos C, Pawlik AJ, Esbrook CL, et al. Early physical and occupational therapy in mechanically ventilated, critically ill patients: a randomised controlled trial. Lancet. 2009;373(9678):1874-82.

33. Lee H, Ju J-W, Oh S-Y, Kim J, Jung CW, Ryu HG. Impact of timing and duration of postoperative delirium: a retrospective observational study. Surgery. 2018;164(1):137-43.

34. Shehabi Y, Riker RR, Bokesch PM, Wisemandle W, Shintani A, Ely EW, et al. Delirium duration and mortality in lightly sedated, mechanically ventilated intensive care patients. Crit Care Med. 2010;38(12):2311-8. 


\title{
Affiliations
}

Tracey Kim ${ }^{1} \cdot$ Christopher Celis $^{1} \cdot$ Andrew Pop $^{1} \cdot$ Kaitlin McArthur $^{1} \cdot$ Thomas Robert Bushell $^{1} \cdot$ Xian Luo-Owen $^{1}$. Lourdes Swentek $^{1} \cdot$ Sigrid Burruss $^{1} \cdot$ Steven Brooks ${ }^{2} \cdot$ David Turay $^{1} \cdot$ Kaushik Mukherjee $^{1}$

\author{
Tracey Kim \\ traceykim87@gmail.com \\ Christopher Celis \\ ccelis@students.llu.edu \\ Andrew Pop \\ apop@students.llu.edu \\ Kaitlin McArthur \\ kmcarthur@students.llu.edu \\ Thomas Robert Bushell \\ tbushell@1lu.edu \\ Xian Luo-Owen \\ xluoowen@1lu.edu \\ Lourdes Swentek \\ Lourdes.Swentek@gmail.com
}

Sigrid Burruss

sburruss@1lu.edu

Steven Brooks

steven.brooks@ttuhsc.edu

David Turay

mrlturay2@yahoo.com

1 Division of Acute Care Surgery, Loma Linda University Medical Center, 11175 Campus Street, CP 21111, Loma Linda, CA 92350, USA

2 Texas Tech University Health Sciences Center, Lubbock, TX, USA 